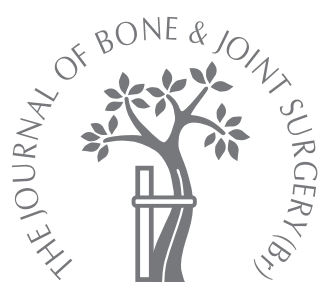
D. A. Simon,
T.-L. Taylor,
G. Bayley,
K.-A. Lalonde
From the Division of
Orthopaedic Surgery,
the University of
Ottawa, Ottawa,
Canada

\title{
Four-limb compartment syndrome associated with the systemic capillary leak syndrome
}

\begin{abstract}
Systemic capillary leak syndrome, or the Clarkson syndrome, is an extremely rare condition in which increased capillary permeability results in a massive shift of fluid into the extravascular space. This is followed rapidly by hypotensive shock, haemoconcentration, and, potentially, substantial oedema of the limbs resulting in an acute compartment syndrome. It is important for orthopaedic surgeons to be aware of this syndrome as our medical colleagues, who initially care for these patients, are less familiar with the diagnosis and the need for emergency management of the associated compartment syndrome should it develop. There have been fewer than 100 cases of this entity reported.

This case report is the first to describe the subsequent development of a compartment syndrome in all four limbs. Clinical vigilance and continuous monitoring of intracompartmental pressure is necessary in these patients in order to help reduce limbthreatening complications.
\end{abstract}

Systemic capillary leak syndrome is an extremely rare condition in which increased capillary permeability results in a massive shift of fluid into the extravascular space, leading to hypotensive shock, haemoconcentration, hypoalbuminaemia, and oedema of the limbs. ${ }^{1}$ Since Clarkson et $\mathrm{al}^{1}$ first described the syndrome in 1960, there have been fewer than 100 cases reported worldwide. The aetiology is generally idiopathic, although it can occur secondarily to a number of other disorders. It is important for the orthopaedic surgeon to recognise this condition, as a potential consequence is an acute compartment syndrome, which has been described in 14 previous cases. The compartment syndrome may be part of the initial presentation or may occur as a result of efforts of resuscitation in an intensive care unit.

We describe the first case of systemic capillary leak syndrome with subsequent development of a compartment syndrome in all four limbs and resulting neuromuscular deficits.

\section{Case report}

A 57-year-old male presented to the emergency department with a history of fatigue, flu-like symptoms and abdominal pain for approximately 36 hours, and mild bilateral calf pain for one week. Two hours prior to presentation he had experienced pre-syncopal episodes and a rapid increase in pain and swelling of the calves. In the preceding month, he had begun a new routine, running on a treadmill for 30 minutes every other day. He denied using steroids, other performance-enhancing drugs or herbal supplements. His past medical history included gastro-oesophageal reflux and hypercholesterolaemia, for which he was taking a statin.

On examination he was in hypotensive shock and had clinical findings consistent with bilateral compartment syndromes of the lower legs. The initial laboratory findings included a haemoglobin level of $220 \mathrm{~g} / \mathrm{l}$, a haematocrit of 0.62 , a white blood cell count of $47.9 \times 10^{9} / \mathrm{L}$, albumin of $15 \mathrm{~g} / \mathrm{l}$, metabolic acidosis and acute renal failure occurred secondary to acute tubular necrosis from rhabdomyolysis (creatine kinase $>8000 \mathrm{U} / \mathrm{l}$ ) and the nephrotoxic effects of liberated myocyte components. A CTangiogram excluded an occlusive vascular event. He was reviewed by the internal medicine and orthopaedic departments. Emergency management included phlebotomy of $600 \mathrm{cc}$ of blood to reduce the hyperviscosity in order to prevent an ischaemic event, as well as aggressive fluid resuscitation and bilateral four compartment fasciotomies of the lower legs.

The initial diagnosis was of a compartment syndrome secondary to hyperviscosity due to polycythaemia. The elevated white blood count was thought to be the result of demargination. The initial differential diagnosis 
Table I. Clinical features of the systemic capillary leak syndrome

Flu-like prodrome: nausea, vomiting, abdominal pain, myalgia
Polydipsia
Dizziness
Hypotension
Generalised/extremity oedema (pitting or non-pitting)
Weight gain
Extremity pain/compartment syndrome/rhabdomyolysis
Pleural/pericardial effusion
Cerebral/pulmonary/macular/epiglottic oedema
Renal dysfunction/failure

Table III. Differential diagnosis for the systemic capillary leak syndrome

Idiopathic
Overwhelming sepsis
Dengue shock syndrome
Hereditary angio-oedema due to $\mathrm{C} 1$ esterase deficiency
Systemic marcocytosis
Chemotherapy
Lymphoma
Sezary syndrome
Carbon monoxide poisoning

\author{
Table II. Laboratory markers in the systemic \\ capillary leak syndrome \\ Polycythaemia/elevated haematocrit (> 55\%) \\ Elevated white blood cell count \\ Low serum protein/albumin \\ Monoclonal immunoglobulinopathy \\ - IgG Kappa \\ - IgG Lambda \\ - $\lg A$ Lambda
}

the JAK2 marker for myeloproliferative disorders. Angiooedema and amyloidosis were also ruled out. The antinuclear antibody, anti-nuclear cytoplasmic antibody and anti-glomerular basement membrane antibody were negative, and the levels of $\mathrm{C} 1$ and $\mathrm{C} 3$ esterase inhibitor were normal. A positive monoclonal (lambda) immunoglobinopathy was present. Ultimately, the diagnosis of the systemic capillary leak syndrome was confirmed, with secondary compartment syndromes in all four limbs.

\section{Discussion}

The hallmark of the systemic capillary leak syndrome is the triad of acute hypovolaemia, hypoalbuminaemia without albuminuria and oedema of the limbs. ${ }^{1-9}$ Up to $70 \%$ of intravascular fluid may shift rapidly into the interstitial space, causing rapid and dramatic rises in the concentrations of marrow elements, which normalise one to four days later when the interstitial fluid is recruited back into the venules. The oedema of the limbs may be severe enough to cause a compartment syndrome.

The syndrome has been described in previously healthy patients ranging in age from three months to 68 years. It appears to have a male-to-female ratio of 1.4:1. ${ }^{2}$ It may occur as an isolated event, but typically relapses. Hypovolaemic shock is a defining feature of the condition. The potential clinical signs and symptoms are shown in Table I, and the laboratory markers in Table II. The detailed pathogenesis and the histological mechanisms are not yet understood, although the majority of patients will have an associated monoclonal immunoglobulinopathy $(\operatorname{IgG}) .^{2-9}$

Most cases of systemic capillary leak syndrome appear to be idiopathic, but different states of disease have been associated with capillary leak (Table III). Treatment is primarily supportive, requiring aggressive fluid resuscitation with adjuvant pressors and/or inotropes to maintain blood pressure and end organ perfusion. ${ }^{2}$ During resuscitation, fluid will continue to shift out of the intravascular space until the leakiness of the capillaries has resolved. This results in increased interstitial pressure in the lower limbs which may lead to a compartment syndrome with rhabdomyolysis as a rare consequence. ${ }^{2,7-9}$ Our case is the first reported incidence of compartment syndromes involving all four limbs. 
Patients with the systemic capillary leak syndrome may lose consciousness due to cerebral hypoperfusion before clinical evidence of a compartment syndrome develops, which can lead to delay in diagnosis and orthopaedic management. Patients may also remain incapacitated during the period of resuscitation, as in our case, which diminishes the ability to diagnose a compartment syndrome in other limbs. ${ }^{10}$ In such circumstances continuous monitoring of intracompartmental pressure can affect a significant reduction in the time to diagnosis and management of the limbs at risk, and therefore reduce permanent sequelae. ${ }^{11}$ This procedure is relatively simple to perform and will allow more rapid diagnosis of a compartment syndrome. Patients with systemic capillary leak syndrome are likely to be cared for initially by medical or intensive care teams comprised of inexperienced junior physicians less familiar with the importance of the timely clinical diagnosis of compartment syndrome and the importance of early surgical decompression.

No benefits in any form have been received or will be received from a commercial party related directly or indirectly to the subject of this article.

\section{References}

1. Clarkson B, Thompson D, Horwith M, Luckey EH. Cyclical edema and shock due to increased capillary permeability. Am J Med 1960;29:193-216.

2. Dhir V, Arya V, Malav IC, et al. Idiopathic systemic capillary leak syndrome (SCLS): case report and systematic review of cases reported in the last 16 years. Intern Med 2007:46:899-904

3. Airaghi L, Montori D, Santambrogio L, Miadonna A, Tedeschi A. Chronic systemic capillary leak syndrome: report of a case and review of the literature. $J$ Intern Med 2000;247:731-5.

4. Dams K, Meersseman W, Verbeken E, Knockaert DC. A 59-year-old man with shock, polycythemia and an underlying paraproteinemia. Chest 2007;132:1393-6.

5. Kawabe S, Saeki T, Tamazaki H, et al. Systemic capillary leak syndrome. Intern Med 2002;41:211-15.

6. Onal H, Aktuglu-Zeybek C, Altun G, et al. Capillary leak syndrome in a 5-monthold infant associated with intractable diarrhoea. Ann Trop Paediatr 2007;27:81-6.

7. Abdul-Ghaffar NU, Farghaly MM, Swamy AS. Acute renal failure, compartment syndrome and systemic capillary leak syndrome complicating carbon monoxide poisoning. J Toxicol Clin Toxicol 1996;34:713-19.

8. Matsumara M, Kakuchi Y, Hamano R, et al. Systemic capillary leak syndrome associated with compartment syndrome. Intern Med 2007;46:1585-7.

9. Sanghavi R, Aneman A, Parr M, Dunlop L, Champion D. Systemic capillary leak syndrome associated with compartment syndrome and rhabdomyolysis. Anaesth Intensive Care 2006;34:388-91

10. Ulmer T. The clinical diagnosis of compartment syndrome of the lower leg: are clinical findings predictive of the disorder? J Orthop Trauma 2002;16:572-7.

11. McQueen MM, Christie J, Court-Brown CM. Acute compartment syndrome in tibial diaphyseal fractures. J Bone Joint Surg [Br] 1996;78-B:95-8. 